\title{
Systèmes à libération contrôlée pH-dépendants de principes actifs hydrophobes à partir d'oléogels
}

\author{
Papa Mady SY ${ }^{1 *}$, Peggy Ngadou NTCHOBAHA ${ }^{1,5}$, Sidy Mouhamed DIENG ${ }^{4}$, \\ Louis Augustin D. DIOUF ${ }^{1}$, Alphonse R. DJIBOUNE ${ }^{1}$, Boucar NDONG ${ }^{2}$, \\ Mamadou SOUMBOUNDOU ${ }^{3}$, Gora MBAYE ${ }^{1}$, Mamadou MBODJ ${ }^{2}$, Guata Yoro $\mathrm{SY}^{5}$ et \\ Mounibé DIARRA ${ }^{1}$
}
${ }^{I}$ Laboratoire de physique pharmaceutique, Faculté de Médecine, de Pharmacie et d'Odontologie, Université Cheikh Anta Diop, Dakar, Sénégal.
${ }^{2}$ Laboratoire de Biophysique Médicale et Médecine Nucléaire, Faculté de Médecine, de Pharmacie et d'Odontologie, Université Cheikh Anta Diop, Dakar, Sénégal.
${ }^{3}$ Service de Biophysique Médicale, UFR Santé, Université de Thiès, Sénégal.
${ }^{4}$ Laboratoire de Pharmacie Galénique et Biopharmacie, UFR Santé, Université de Thiès, Sénégal.
${ }^{5}$ Laboratoire de Pharmacologie, Faculté de Médecine, de Pharmacie et d'Odontologie, Université Cheikh Anta Diop, Dakar, Sénégal.

"Auteur correspondant ; E-mail : papamady.sy@ucad.edu.sn, mounibe.diarra@ucad.edu.sn; Tel. : +221 775601528, +221775022172, + 221338652341

\begin{abstract}
Received: 05-02-2021
Accepted:14-04-2021

Published: 30-04-2021

Les rhumatismes inflammatoires chroniques sont une cause importante d'invalidité dans le monde entier. De ce fait, les affections rhumatismales chroniques font peser une lourde charge sociale et économique sur toutes les sociétés, pas seulement sur celles où l'espérance de vie est élevée. L'objectif principal de ce travail était d'étudier le profil de libération pH-dépendante de principes actifs hydrophobes à partir d'oléogels oraux et/ou cutanés. La formulation des oléogels a été réalisée selon une méthode sol-gel, reproductible à grande échelle. La caractérisation et le suivi dans le temps ont montré une bonne stabilité des oléogels. Les valeurs de pH des oléogels étaient globalement acides (entre 4,3 et 5,8) et dépendaient de la quantité de gélifiant utilisée. Les études de libération du kétoprofène, principe actif hydrophobe, en fonction du $\mathrm{pH}$ des milieux de dissolution ont montré

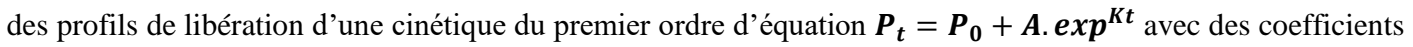
de détermination proches de 1 (milieux à $\mathrm{pH}$ égal à 1,2 et 5,5). Une meilleure libération du kétoprofène a été obtenue dans un milieu intestinal simulé (pH égal à 6,8$)$ pour les formulations qui présentaient déjà une saturation en milieu gastrique simulé ( $\mathrm{pH}$ égal à 1,2 ). Cette étude qui a permis de formuler, d'évaluer et de modéliser le profil de libération du kétoprofène à partir d'oléogels peut constituer une étape importante dans un objectif de souveraineté thérapeutique des pays d'Afrique subsaharienne notamment le Sénégal.
\end{abstract}

\section{RESUME}

(C) 2021 International Formulae Group. All rights reserved.

Mots clés : Oléogels, rhumatismes inflammatoires chroniques, kétoprofène, libération contrôlée, pH-dépendant. 


\title{
pH-dependent controlled release systems of hydrophobic active pharmaceutical ingredients from oleogels
}

\begin{abstract}
Chronic inflammatory rheumatism is a major cause of disability around the world. As a result, chronic rheumatic diseases place a heavy social and economic burden on all societies, not just those with high life expectancy. The main objective of this work was to control the $\mathrm{pH}$-dependent release of hydrophobic active pharmaceutical ingredients from oral and / or skin oleogels. The formulation of the oleogels was carried out using a sol-gel large-scale reproducible method. Characterization and monitoring over time have shown good stability of the oleogels. The $\mathrm{pH}$ values of the oleogels were overall acid (between 4.3 and 5.8) and depended on the amount of gelling agent used. The release studies of ketoprofen, a hydrophobic active pharmaceutical ingredient, as a function of the $\mathrm{pH}$ of the dissolution media have shown release profiles of first-order kinetics of equation $\boldsymbol{P}_{\boldsymbol{t}}=\boldsymbol{P}_{\mathbf{0}}+\boldsymbol{A} \cdot \boldsymbol{e x p}^{\boldsymbol{K} \boldsymbol{t}}$ with coefficients of determination close to 1 (media at pH equal to 1.2 and 5.5). Better release of ketoprofen was obtained in simulated intestinal medium (pH equal to 6.8) for formulations which already exhibited saturation in simulated gastric medium ( $\mathrm{pH}$ equal to 1.2). This study, which made it possible to formulate, evaluate and model the release profile of ketoprofen from oleogels, may constitute an important step in an objective of therapeutic sovereignty of the countries of sub-Saharan Africa, particularly Senegal.

(C) 2021 International Formulae Group. All rights reserved.
\end{abstract}

Keywords: oleogels - chronic inflammatory rheumatism - ketoprofen - controlled release - pH-dependent.

\section{INTRODUCTION}

Les pathologies rhumatismales sont des causes majeures d'inconfort et d'incapacité. Elles entraînent des douleurs articulaires importantes, une limitation fonctionnelle et une qualité de vie altérée (Jeandel et Roux, 2002; Seidel et al., 2015). La prévalence de celles-ci augmente avec l'âge. Ces pathologies sont associées à d'autres affections concomitantes, telles que le diabète et les maladies cardiovasculaires, ce qui complique encore leurs traitements (Rother et Conaghan, 2013). Les traitements pharmacologiques, non pharmacologiques et chirurgicaux actuels, visent à soulager la douleur et la raideur articulaire et à maintenir ou améliorer la fonction physique (Derry et al., 2015; Rother et Conaghan, 2013). Parmi ces traitements, l'application topique et/ ou per os d'AntiInflammatoires Non Stéroïdiens (AINS) contenant du kétoprofène, de l'ibuprofène, du diclofénac sous forme de pommades, de crèmes ou de gels, occupe une place importante. Les gels conservent une plus grande stabilité et une libération plus rapide des substances actives que les crèmes ou les pommades (Verma et al., 2013;
Mazurkeviciute et al., 2017). Un gel est une solution ou une suspension colloïdale qui subit une transformation physique ou chimique conduisant à un état semi-solide tout en conservant une grande partie du solvant à l'intérieur de sa structure (Mazurkeviciute et al., 2017; Dantas et al., 2016; Iwanaga et al., 2012). Divers agents gélifiants sont utilisés dans la production de gels : des polymères naturels, des dérivés de cellulose semisynthétiques et des polymères synthétiques (Osada et al., 2004; Rehman et Zulfakar, 2014). Il existe plusieurs bases de classifications des gels : selon leur origine, avec les gels naturels ou synthétiques; selon le type de liaison de la matrice polymère, avec les gels chimiques ou physiques; selon la matière contenue dans la matrice polymère, avec les hydrogels, les organogels ou oléogels (Osada et al., 2004; Rehman et Zulfakar, 2014). Les oléogels sont des systèmes semi-solides obtenus avec un organo-gélateur et un liquide hydrophobe. Ils font l'objet de nombreuses recherches au cours des dernières années et qui pourraient jouer un rôle important en tant que réservoir pour la libération des principes actifs (PA) au niveau cutané et par voie générale (Esch et Feringa, 
2000). La formulation de ces systèmes hydrophobes nécessite une certaine dextérité (Almeida et Bahia, 2006). L'objectif de ce travail était de formuler des oléogels et d'évaluer la libération du kétoprofène à partir de ces oléogels.

\section{MATERIEL ET METHODES Matériel}

La gélatine, l'hydroxyde de sodium, le phosphate de potassium monobasique et le phosphate de potassium dibasique ont été obtenus auprès de Sigma-Aldrich. La phase huileuse utilisée tout au long de l'étude est une huile de paraffine officinale. La phase aqueuse utilisée est de l'eau distillée. Le kétoprofène a été obtenu auprès des laboratoires FAGRON S.A (Saint-Denis, France). Les filtres à seringue Acrodisc avec membrane en nylon et tube à membrane de dialyse (tube à membrane poreuse moléculaire Spectra / Por MWCO 12$14 \mathrm{kDa}$ ) ont été obtenus respectivement auprès des laboratoires PALL Life Sciences et Spectrum (USA). Tous les produits chimiques étaient de qualité analytique.

\section{Méthodes}

\section{Formulation des oléogels}

La formulation des oléogels a été réalisée en faisant fondre la cire avec une petite quantité d'huile de paraffine (température inférieure à $60{ }^{\circ} \mathrm{C}$ ). Dans ce mélange, une quantité de kétoprofène exactement pesée, est incorporée avant l'ajout de la quantité d'huile restante, tout en agitant pour homogénéiser. Parallèlement, la solution polymérique est préparée en mélangeant l'eau avec de la gélatine à chaud. Enfin, les deux préparations sont mélangées en agitant et en chauffant (60 $\left.{ }^{\circ} \mathrm{C}\right)$ pendant $1 \mathrm{~min} 30 \mathrm{~s}$. L'oléogel obtenu est répartie dans des tubes à essai puis refroidie à température ambiante dans un bac contenant de l'eau tiède.

Le Tableau 1 présente les différentes quantités de produits utilisés pour la préparation des oléogels.
Caractérisation physico-chimique des oléogels

Examen macroscopique

Après formulation des oléogels, nous avons procédé à l'examen macroscopique. Les oléogels sont laissés verticalement au repos à l'abri de la lumière et à température ambiante dans des tubes à essai. Le but de cette inspection visuelle est de mettre en évidence certains phénomènes d'instabilité de la formulation, tels que la formation de dépôt (saturation), la séparation des phases, la formation d'agrégat...

Mesures de la conductivité et du $\mathrm{pH}$

La résistance électrique et le $\mathrm{pH}$ des oléogels ont été mesurés à l'aide d'un conductimètre et d'un $\mathrm{pH}$-mètre Schott Geräte muni d'une électrode de mesure. Le temps de lecture est fixé à deux minutes après l'introduction de la cellule.

\section{Etudes de libération in vitro du Kétoprofène}

Les profils de libération in vitro ont été obtenus en utilisant une membrane de dialyse (12000 - 14000 Da). Le tube de dialyse contenant $2 \mathrm{~g}$ d'oléogels et $2 \mathrm{ml}$ du milieu de dissolution, a été introduit dans le milieu de libération in vitro contenant $250 \mathrm{ml}$ du milieu de dissolution sous agitation à 100 tours/min. Ce milieu de dissolution est constitué d'une solution tampon à $\mathrm{pH} 5,5$ simulant le $\mathrm{pH}$ cutané et d'une solution tampon à $\mathrm{pH} 1,2$ et 6,8 simulant le $\mathrm{pH}$ du tractus gastro-intestinal. Les assemblages entiers ont été maintenus à une température de $37^{\circ} \mathrm{C} \pm 1$. A intervalle de 15 minutes, les échantillons de solution ont été prélevés et analysés par spectrophotométrieUV visible. Les conditions «sink» ont été maintenues en remplaçant chaque fois $5 \mathrm{ml}$ du milieu de libération par un milieu frais à chaque point d'échantillonnage. Le pourcentage de PA libéré, a été obtenu à partir de la quantité de kétoprofène initialement présente dans la formulation, comparée à celle mesurée dans le milieu de libération. Toutes les mesures ont été réalisées trois fois. 
Tableau 1 : Quantités de produits utilisés pour la formulation des oléogels contenant du kétoprofène.

\begin{tabular}{lcccccccccc} 
& T1 & T2 & T3 & T4 & T5 & T6 & T7 & T8 & T9 & T10 \\
\hline Huile de paraffine (g) & 15,4 & 15,4 & 15,4 & 15,4 & 15,4 & 15,4 & 15,4 & 15,4 & 15,4 & 15,4 \\
& & & & & & & & & & \\
\hline Cire d'abeille (g) & 2,5 & 2,5 & 2,5 & 2,5 & 2,5 & 2,5 & 2,5 & 2,5 & 2,5 & 2,5 \\
& & & & & & & & & & \\
\hline Gélatine (g) & 0,47 & 0,43 & 0,39 & 0,35 & 0,31 & 0,27 & 0,23 & 0,19 & 0,15 & 0,11 \\
\hline Eau (g) & 10,88 & 10,92 & 10,96 & 11 & 11,04 & 11,08 & 11,12 & 11,16 & 11,20 & 11,24 \\
& & & & & & & & & & \\
\hline Kétoprofène (g) & 0,75 & 0,75 & 0,75 & 0,75 & 0,75 & 0,75 & 0,75 & 0,75 & 0,75 & 0,75 \\
\end{tabular}

\section{RESULTATS}

L'étude des paramètres physicochimiques et analytiques des différents oléogels, conservés à l'abri de la lumière et à température ambiante pendant un mois, a permis de suivre leur évolution en fonction du temps.

\section{Caractérisation physico-chimique Examen macroscopique}

Après formulation, nous avons obtenu pour l'ensemble des gels, un aspect blanchâtre, visqueux avec une odeur de cire d'abeille (Figure 1).

Ces oléogels peuvent être classés en deux catégories : homogène et biphasique (Tableau 2).

La majorité des tubes a présenté un aspect homogène et stable sans formation de dépôts ni séparation de phase pendant toute la durée de conservation. Cependant, le tube T3 a présenté un aspect biphasique. Ceci peut s'expliquer en partie par le fait que les gélifiants n'ont pas réussi à constituer une charpente qui retient la phase continue et se sont accumulés au fond du tube.

\section{Conductivité}

$\mathrm{Au}$ bout de 24 heures après formulation, la mesure de la conductivité a donné des valeurs quasi-nulles (environ 0,00 $\mathrm{mS} . \mathrm{cm}^{-1}$ ) dans tous les tubes. Ces valeurs ont été confirmées durant toute la durée de stockage. En effet, la phase liquide est continue et la phase solide constitue une charpente qui emprisonne la phase continue. Dans les formulations d'oléogels, la phase continue est constituée par l'huile de paraffine, or la conductivité des huiles est quasi-nulle, ce qui justifie les résultats de conductivité obtenues.

\section{Valeurs du pH}

La Figure 2 montre l'évolution du $\mathrm{pH}$ des gels en fonction de la quantité de gélifiant. Le $\mathrm{pH}$ des formulations était acide durant toute la durée de conservation (entre 4,3 et 5,8). Ces valeurs de $\mathrm{pH}$ étaient fortement liées à la quantité de gélatine incorporée. En effet, pour des quantités de gélifiant comprises entre 0,1 et $0,2 \mathrm{~g}$, les valeurs de $\mathrm{pH}$ étaient comprises entre 5,7 et 5,8 , entre 5,1 et 5,4 lorsque les quantités de gélifiants passaient de 0,2 à $0,4 \mathrm{~g}$. Enfin, les oléogels étaient plus acides ( $\mathrm{pH}$ entre 4,3 et 4,8 ) quand on dépassait $0,4 \mathrm{~g}$ de gélifiant. Ces résultats se reproduisaient dans tous les tubes.

\section{Etudes de libération in vitro du} kétoprofène

Profils de libération du kétoprofène dans un milieu gastrique simulé suivi d'une étude dans un milieu intestinal simulé

La Figure 3 montre des profils de libération du kétoprofène dans un milieu gastrique simulé. Ces profils ont été modélisés selon une cinétique du premier ordre d'équation :

$$
P_{t}=P_{0}+A \cdot \exp ^{K t}
$$

La modélisation des profils de libération du kétoprofène à partir des oléogels selon une cinétique du premier ordre, avec des coefficients de détermination $\left(\mathrm{R}^{2}\right)$ proches de 
1, montre que la concentration résiduelle du PA dans la matrice conditionnait la libération. En milieu gastrique simulé, du fait de la faible solubilité du kétoprofène (PA appartenant à la classe II du système de classification biopharmaceutique), sa concentration de saturation était rapidement atteinte. Ce qui faisait que les pourcentages de libération étaient inférieurs à environ $30 \%$.

Le Tableau 3 présente les paramètres de modélisation des profils de libération dans un milieu gastrique simulé ( $\mathrm{pH}$ égal à 1,2). Nous remarquons que les coefficients de détermination $\left(\mathrm{R}^{2}\right)$ sont proches de 1 . Ce qui montre une très bonne corrélation entre les profils obtenus et ceux d'une cinétique du premier ordre.

La Figure 4 montre des profils de libération du kétoprofène dans un milieu gastrique simulé suivi d'une étude dans un milieu intestinal simulé.

En augmentant les valeurs du $\mathrm{pH}$ (Milieu intestinal simulé), une meilleure libération du kétoprofène était obtenue. Ce qui montre que la libération du kétoprofène est $\mathrm{pH}$ dépendante et en jouant sur le $\mathrm{pH}$ de la formulation d'oléogels (notamment la quantité de gélifiant), on pourrait améliorer sa biodisponibilité.

Profils de libération du kétoprofène dans un milieu simulant le $\mathrm{pH}$ cutané $(\mathrm{pH}=5,5)$

La Figure 5 montre des profils de libération du kétoprofène dans un milieu simulant le $\mathrm{pH}$ cutané $(\mathrm{pH}=5,5)$, modélisés selon une cinétique du premier ordre d'équation : $\boldsymbol{P}_{t}=\boldsymbol{P}_{0}+\boldsymbol{A} \cdot \boldsymbol{e x p}^{K t}$

Par rapport à un milieu simulant le $\mathrm{pH}$ cutané (pH égal à 5,5), la modélisation des profils de libération selon une cinétique du premier ordre a donné des coefficients de détermination $\left(\mathrm{R}^{2}\right)$ proches de 1 . Ce qui montre également dans ce milieu comme le milieu gastrique simulé, la libération était fonction de la concentration résiduelle de kétoprofène dans la matrice.

Le Tableau 4 présente les paramètres de modélisation des profils de libération.



Figure 1 : Aspect macroscopique du tube T5 après formulation.

Tableau 2 : Différents aspects des oléogels après inspection visuelle.

\begin{tabular}{lc}
\hline Observations & Tubes \\
\hline Oléogel homogène & $\mathrm{T} 1, \mathrm{~T} 2, \mathrm{~T} 4, \mathrm{~T} 5, \mathrm{~T} 6, \mathrm{~T} 7, \mathrm{~T} 8, \mathrm{~T} 9, \mathrm{~T} 10$ \\
\hline Oléogel biphasique & $\mathrm{T} 3$ \\
\hline
\end{tabular}




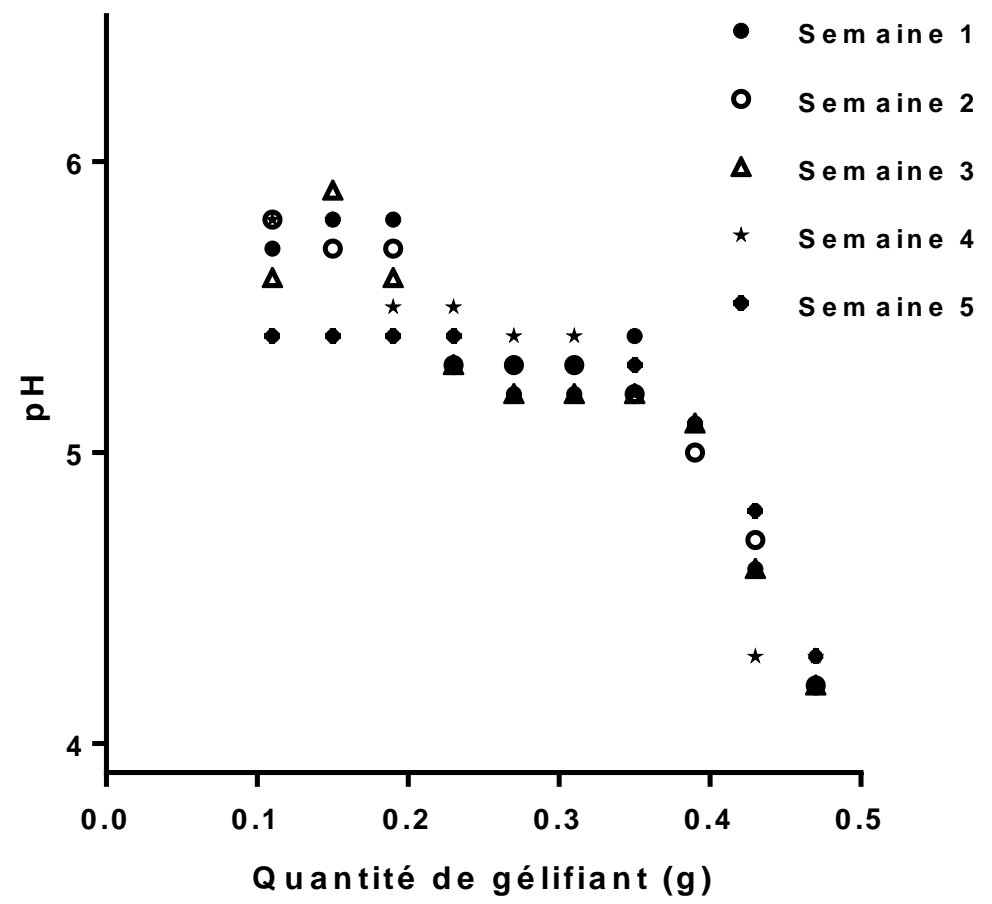

Figure 2 : Evolution du pH des formulations pendant 1 mois selon la quantité de gélifiant utilisée.

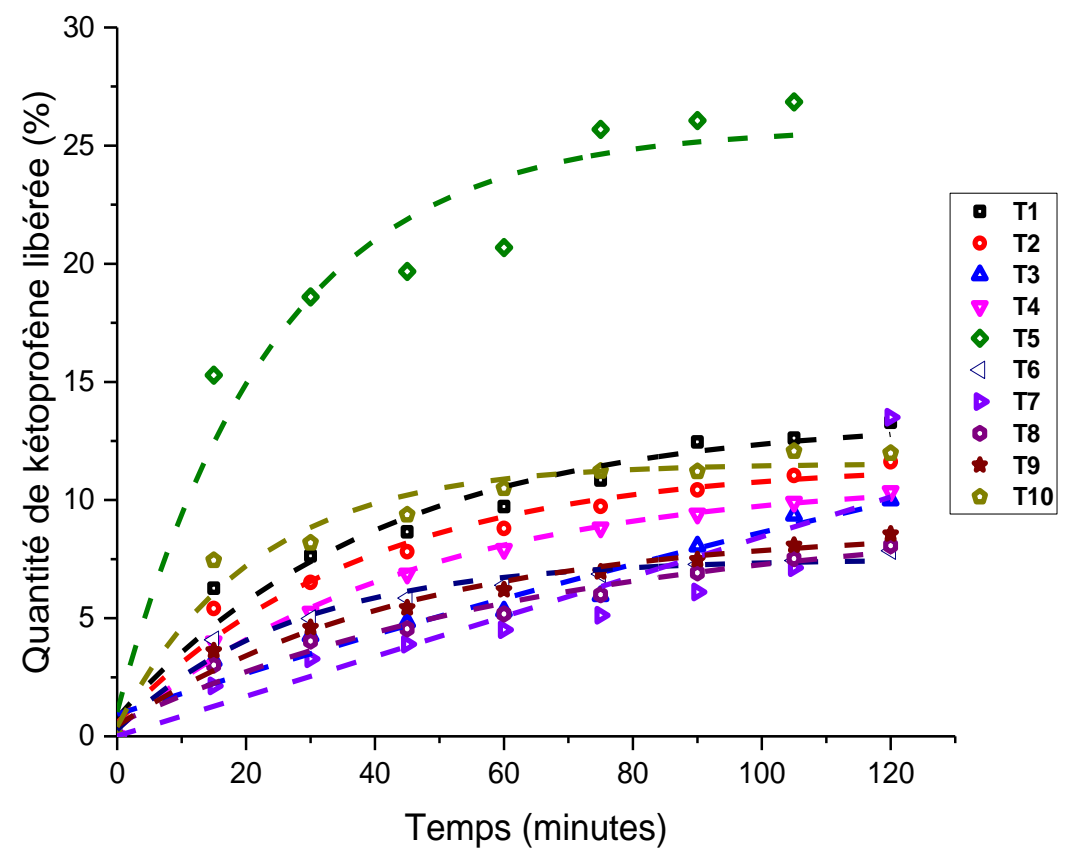

Figure 3 : Profils de libération du kétoprofène dans un milieu gastrique simulé à pH égal à 1,2. 
Tableau 3 : Paramètres de modélisation des profils de libération dans un milieu à pH égal à 1,2 selon une cinétique du premier ordre.

\begin{tabular}{cccccccc} 
& \multicolumn{2}{c}{$\mathbf{P}_{\mathbf{0}}$} & \multicolumn{2}{c}{$\mathbf{A}$} & \multicolumn{2}{c}{ K } & $\mathbf{R}^{\mathbf{2}}$ \\
\cline { 2 - 8 } & Valeur & Ecart-type & Valeur & Ecart-type & Valeur & Ecart-type \\
\hline T1 & 21,47 & 2,1 & $-20,59$ & 1,9 & $-0,015$ & 0,003 & $\mathbf{0 , 9 7 7}$ \\
\hline T2 & 15,8 & 0,7 & $-15,37$ & 0,65 & $-0,019$ & 0,002 & $\mathbf{0 , 9 9 1}$ \\
\hline T3 & $-57,42$ & 179,82 & 56,005 & 177,83 & 0,0032 & 0,008 & $\mathbf{0 , 8 5 5}$ \\
\hline T4 & 15,92 & 0,2 & $-15,84$ & 0,48 & $-0,07$ & 0,005 & $\mathbf{0 , 9 9 2}$ \\
\hline T5 & 14,97 & 0,65 & $-14,99$ & 0,59 & $-0,01$ & 0,001 & $\mathbf{0 , 9 9 6}$ \\
\hline T6 & 32,5 & 3,8 & $-32,1$ & 3,65 & $-0,006$ & 0,001 & $\mathbf{0 , 9 9 7}$ \\
\hline T7 & 31,45 & 2,02 & $-31,17$ & 1,92 & $-0,007$ & $7,72^{\mathrm{E}}-4$ & $\mathbf{0 , 9 9 8}$ \\
\hline T8 & 9,12 & 0,69 & $-8,65$ & 0,69 & $-0,021$ & 0,0049 & $\mathbf{0 , 9 6 3}$ \\
\hline T9 & 13,42 & 0,51 & $-13,3$ & 0,46 & $-0,011$ & $7,78^{\mathrm{E}}-4$ & $\mathbf{0 , 9 9 8}$ \\
\hline T10 & 6,62 & 0,23 & $-6,41$ & 0,39 & $-0,039$ & 0,005 & $\mathbf{0 , 9 7 1}$ \\
\hline
\end{tabular}

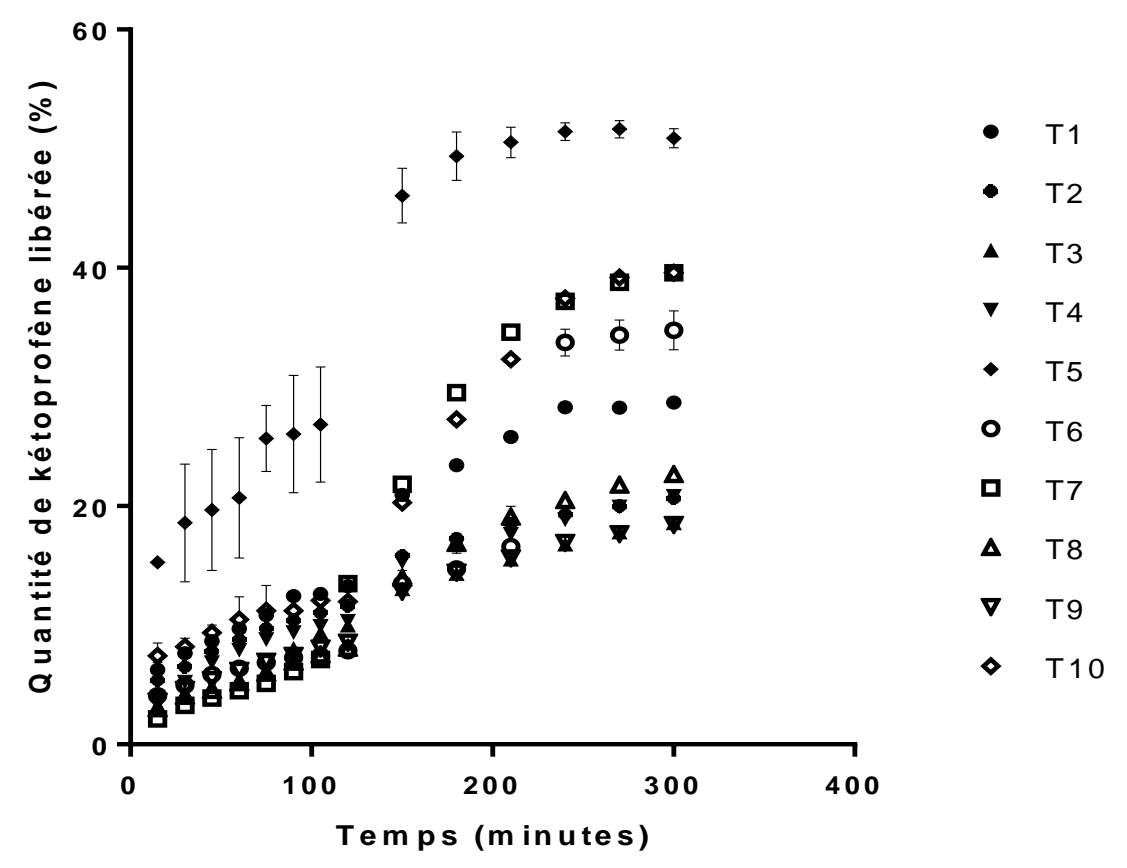

Figure 4 : Profils de libération du kétoprofène dans un milieu gastrique simulé pendant 2 heures suivie d'une libération dans un milieu intestinal simulé pendant 3 heures. 


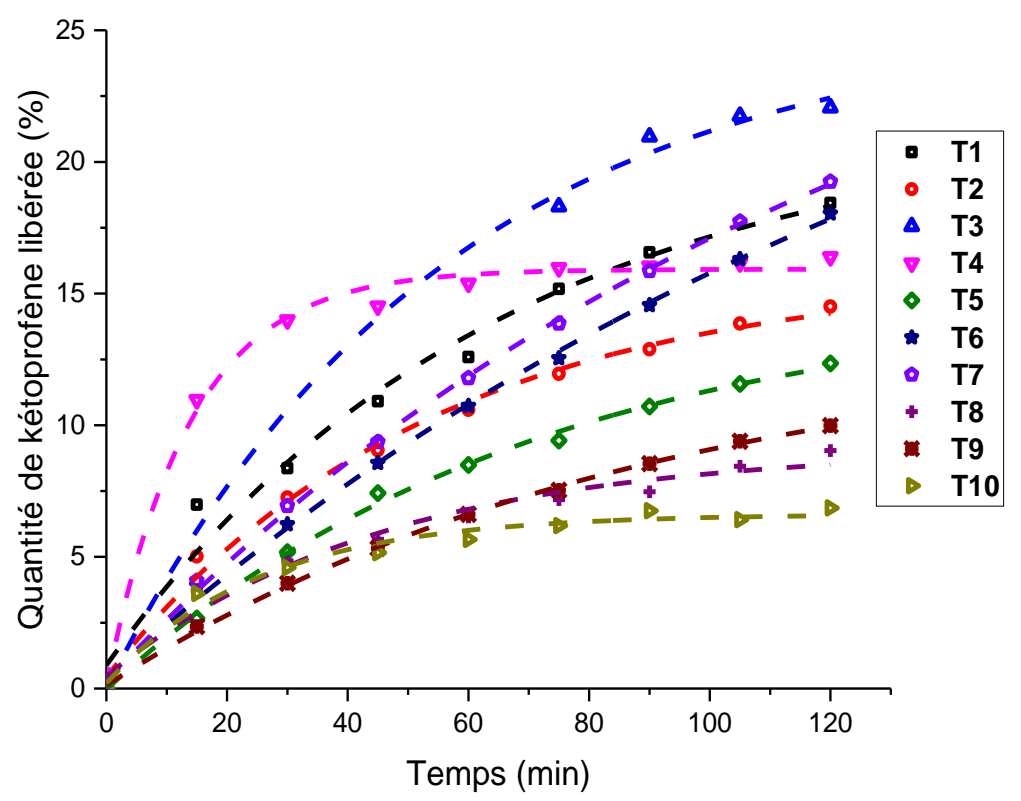

Figure 5 : Profils de libération du kétoprofène dans un milieu simulant le pH cutané $(\mathrm{pH}=5,5)$.

Tableau 4 : Paramètres de modélisation des profils de libération dans un milieu à pH égale à 5,5 selon une cinétique du premier ordre.

\begin{tabular}{cccccccc} 
& \multicolumn{3}{c}{ P0 } & \multicolumn{3}{c}{ A } & \multicolumn{2}{c}{ K } & \multirow{2}{*}{ Valeur } & Ecart-type & Valeur & Ecart-type & Valeur & Ecart-type & $\mathbf{R}^{2}$ \\
\cline { 2 - 6 } T1 & 13,4 & 1,007 & $-12,64$ & 1,098 & $-0,02$ & 0,005 & $\mathbf{0 , 9 5 1}$ \\
\hline T2 & 11,57 & 0,677 & $-10,99$ & 0,776 & $-0,02$ & 0,005 & $\mathbf{0 , 9 6 5}$ \\
\hline T3 & 29,71 & 41,633 & $-28,77$ & 41,235 & $-0,003$ & 0,005 & $\mathbf{0 , 9 3 6}$ \\
\hline T4 & 10,91 & 0,449 & $-10,61$ & 0,453 & $-0,022$ & 0,002 & $\mathbf{0 , 9 8 9}$ \\
\hline T5 & 25,76 & 1,694 & $-24,73$ & 2,57 & $-0,04$ & 0,01 & $\mathbf{0 , 9 2 9}$ \\
\hline T6 & 7,51 & 0,281 & $-7,24$ & 0,443 & $-0,03$ & 0,005 & $\mathbf{0 , 9 7}$ \\
\hline T7 & 12938,16 & $2,14 \mathrm{E} 7$ & $-1,29 \mathrm{E} 4$ & $2,14 \mathrm{E} 7$ & $-6,5 \mathrm{E}-6$ & 0,01 & $\mathbf{0 , 7 7 3}$ \\
\hline T8 & 9,36 & 1,352 & $-8,82$ & 1,221 & $-0,014$ & 0,004 & $\mathbf{0 , 9 6}$ \\
\hline T9 & 8,78 & 0,613 & $-8,33$ & 0,617 & $-0,022$ & 0,004 & $\mathbf{0 , 9 6 8}$ \\
\hline T10 & 11,54 & 0,451 & $-11,13$ & 0,865 & $-0,04$ & 0,008 & $\mathbf{0 , 9 5 3}$ \\
\hline
\end{tabular}




\section{DISCUSSION}

Les principaux résultats obtenus en ce qui concerne les propriétés physiques, ont montré que macroscopiquement la majorité des tubes a présenté un aspect homogène et stable sans formation de dépôts ni séparation de phases pendant toute la durée de conservation. Cependant, le tube T3 a présenté un aspect biphasique. Ceci peut s'expliquer en partie par le fait que les gélifiants se sont accumulés au fond du tube. En effet, la quantité de gélifiants utilisée étant assez importante dans certains tubes (par rapport à la quantité d'eau dans la phase polymérique), il y'a eu un potentiel phénomène de saturation. Cependant, l'absence ou la présence de changement perçu à l'œil nu ne présage pas de la stabilité des gels. En outre, la nature de la liaison définit le type de gel. En effet, l'existence de liaisons fortes, comme les liaisons covalentes permet d'avoir des gels chimiques caractérisés par leur irréversibilité, cependant des liaisons de faible énergie comme des liaisons hydrogène et Van der Waals favorisent la formation de gels physiques. Pour ce qui est de cette étude, nous avons obtenu des gels physiques qui sont caractérisés par leur réversibilité (Philip et al., 2000; Pinhas, 2016; Martinez et al., 2019).

La conductivité des gels est quasi-nulle dans l'ensemble des tubes. Pour les gels lipophiles caractérisés par une faible polarité, la conductivité doit être proche de zéro. En effet, la phase liquide est continue et la phase solide constitue une charpente qui emprisonne la phase continue. Dans notre formulation, la phase continue est constituée par l'huile de paraffine, or la conductivité des huiles est quasi-nulle, ce qui justifie les résultats de conductivité obtenues. Des études ont montré que la conductivité des émulsions E/H doit être inférieure ou égale à $0,01 \mathrm{mS} . \mathrm{cm}^{-1}$ et une valeur constante de la conductivité dans le temps, est un critère déterminant de la stabilité (Dantas et al., 2016; Bar-Cohen, 2004; Sagiri et al., 2014; Rehman et Zulfakar, 2014).

En ce qui concerne le $\mathrm{pH}$, les résultats obtenus ont indiqué un caractère acide pour l'ensemble des tubes, avec des valeurs qui oscillaient entre 4,3 et 5,8. Nous avons également constaté que les valeurs du $\mathrm{pH}$ étaient fortement influencées par la quantité de gélifiant. Ainsi, pour des quantités de gélifiant comprises entre 0,1 et $0,2 \mathrm{~g}$, les valeurs de $\mathrm{pH}$ étaient comprises entre 5,7 et 5,8, entre 5,1 et 5,4 lorsque les quantités de gélifiants passaient de 0,2 à $0,4 \mathrm{~g}$. Enfin, les oléogels étaient plus acides (pH entre 4,3 et 4,8 ) quand on dépassait $0,4 \mathrm{~g}$ de gélifiant. Ces résultats se reproduisaient dans tous les tubes. Ces valeurs de $\mathrm{pH}$ acide confirment les possibilités d'application cutanée des gels formulés, étant donné que le $\mathrm{pH}$ de la peau a une valeur comprise entre 5,4 et 5,9 en dehors des aisselles, des espaces interdigitaux et les orteils (Balata et al., 2014; Morales et al., 2009). Elles offrent également la possibilité d'administration par voie orale.

L'un des objectifs de notre étude était d'évaluer la libération in vitro du kétoprofène pour les différentes formulations dans les milieux simulant respectivement le milieu gastrique, intestinal et cutané. Il ressort de notre étude que le kétoprofène est bien libéré du réseau macromoléculaire et diffuse dans le milieu de libération à des proportions variables. Ainsi, les pourcentages libérés en kétoprofène atteignaient une concentration de saturation après 120 minutes d'étude. Ce qui faisait que les pourcentages libérés n'atteignaient pas $30 \%$ de la charge initiale en milieu acide $(\mathrm{pH} 1,2$ et 5,5). Ainsi, plusieurs profils de libération en fonction du temps ont été obtenus. Ces profils ont été modélisés suivant une cinétique du premier ordre d'équation $\boldsymbol{P}_{\boldsymbol{t}}=\boldsymbol{P}_{\mathbf{0}}+\boldsymbol{A} \cdot \boldsymbol{e} \boldsymbol{x} \boldsymbol{p}^{\boldsymbol{K} t}$ avec des coefficients de détermination, proches de 1. Ce qui fait que la quantité de Kétoprofène libérée était fonction de sa quantité résiduelle dans les oléogels. Dans le milieu intestinal simulé à $\mathrm{pH}=6,8$, nous avons trouvé un pourcentage de libération plus important à partir des oléogels qui présentaient déjà une saturation en milieu acide $(\mathrm{pH}=1,2)$. En effet, le kétoprofène présente une libération fortement influencée par le $\mathrm{pH}$ du milieu (Figure 4) et en jouant sur la quantité de gélifiant utilisée, on peut augmenter le $\mathrm{pH}$ du milieu et améliorer sa biodisponibilité (Sy et al., 2018). 


\section{Conclusion}

$\mathrm{Au}$ terme de nos travaux, il ressort objectivement que les oléogels à base de gélatine contenant du kétoprofène à $2,5 \%$, ont présenté des propriétés physico-chimiques optimales durant toutes leurs périodes d'observation à l'exception du tube T3 pour lequel une séparation des phases a été notée. Par ailleurs, des quantités importantes de principe actif ont diffusé dans le milieu de dissolution après libération à travers le réseau macromoléculaire du gel et passage à travers la membrane de dialyse. Cette capacité des oléogels à libérer les substances actives a été modélisée selon une cinétique du premier ordre d'équation $\boldsymbol{P}_{\boldsymbol{t}}=\boldsymbol{P}_{\mathbf{0}}+\boldsymbol{A} \cdot \boldsymbol{e x p}^{\boldsymbol{K} \boldsymbol{t}}$ avec des coefficients de détermination $\left(\mathrm{R}^{2}\right)$ proches de 1. Cette modélisation a pour intérêt de pouvoir prédire la quantité de kétoprofène qui sera libérée en fonction du temps. Cette étude constitue une étape importante et préliminaire dans un objectif de souveraineté de l'industrie pharmaceutique locale notamment de production des formes orales ou à usage externe, en Afrique.

\section{CONFLIT D'INTERETS}

Les auteurs déclarent qu'ils n'ont pas de conflit d'intérêts.

\section{CONTRIBUTIONS DES AUTEURS}

M-D et PM-S ont conçu l'étude ; PM-S, $\mathrm{PN}-\mathrm{N}$ et SM-D ont réalisé toutes les expériences ; LAD-D, AR-D, G-M, PM-S et M-D ont analysé les données ; PM-S et PN-N ont rédigé l'article avec la contribution de tous les auteurs ; M-S, B-N, M-M, G-M, GY-S et M-D ont apporté leurs corrections et suggestions au manuscrit.

\section{REMERCIEMENTS}

Nous exprimons nos remerciements au Professeur Guata Yoro Sy et à toute l'équipe du Laboratoire de Pharmacologie et Pharmacodynamie de la faculté de Médecine, de Pharmacie et d'Odontologie de l'Université Cheikh Anta Diop de Dakar (Sénégal).

\section{REFERENCES}

Mazurkeviciute A, Ramanaukiene K, Ivaskiene M, Grigonis A, Briedis V.
2017. Modelling and biopharmaceutical evaluation of ciclopirox-olamine gels. Acta Poloniae Pharmaceutica - Drug Research (Polish Pharmaceutical society), 74(2): 543-549.

Almeida IF, Bahia MF. 2006. Evaluation of the physical stability of two oleogels. International Journal of Pharmaceutics, 327(1-2): 73-77. https://doi.org/10.1016/j.ijpharm.2006.0 7.036

Balata G, Nahas HME, Radwan S. 2014. Propolis organogel as a novel topical delivery system for treating wounds. Drug Delivery, 21(1): 55-61. DOI: https://doi.org/10.3109/10717544.2013.8 47032

Bar-Cohen Y. 2004. Electroactive Polymer (EAP) Actuators as Artificial Muscles: Reality, Potential, and Challenges (2nd edn). SPIE Press: Bellingham, USA. DOI: https://doi.org/10.1117/3.547465

Dantas MGB, Reis SAGB, Damasceno CMD, Rolim LA, Rolim-Neto PJ, Carvalho FO, Quintans-Junior LJ, Almeida JRGS. 2016. Development and Evaluation of Stability of a Gel Formulation Containing the Monoterpene Borneol. The Scientific World Journal, 2016: 1-4. DOI: https://doi.org/10.1155/2016/7394685

Pinhas MD. 2016. Oleogels: a promising tool for delivery of hydrophobic bioactive. Ther. Deliv., 7: 7- 9. DOI: https://doi.org/10.4155/tde.15.83

Derry S, Moore RA, Gaskell H, McIntyre M, Wiffen PJ. 2015. Topical NSAIDs for acute musculoskeletal pain in adults. Cochrane Database of Systematic Reviews, 6: CD007402. DOI: https://doi.org/10.1002/14651858.CD00 7402.pub3

Esch VJH, Feringa BL. 2000. New Functional Materials Based on Self-Assembling Organogels: From Serendipity towards Design. Angewandte Chemie, 39(13): 2263-2266.

DOI:

https://doi.org/10.1002/15213773(20000703)39:13\%3C2263::AIDANIE2263\%3E3.0.CO;2-V

Iwanaga K, Kawai M, Miyazaki M, Kakemi M. 2012. Application of organogels as oral controlled release formulations of 
hydrophilic drugs. International Journal of Pharmaceutics, 436(1-2): 869-72. DOI:

https://doi.org/10.1016/j.ijpharm.2012.0 6.041 .

Jeandel P, Roux H. 2002. Épidémiologie des affections rhumatologiques en Afrique subsaharienne. Revue du Rhumatisme, 69(8): 764-776. DOI: https://doi.org/10.1016/s11698330(02)00389-7.

Martinez RM, Rosado C, Velasco MVR, Lannes SCS, Baby AR. 2019. Main features and applications of organogels in cosmetics. International Journal of Cosmetic Science, 41(2): 109-117. DOI: https://doi.org/10.1111/ics.12519

Morales ME, Gallardo V, Clarés B, García MB, Ruiz MA. 2009. Study and description of hydrogels and organogels as vehicles for cosmetic active ingredients. Journal of Cosmetic Science, 60(6): 627-636. DOI: https://doi.org/10.1111/j.14682494.2010.00580_4.X

Osada Y, Gong JP, Tanaka Y. 2004. Polymer Gels. Journal of Macromolecular Science, Part C: Polymer Reviews, 44(1): 87-112.

DOI: https://doi.org/10.1081/MC-120027935

Philip J, Bonakdar L, Poulin P, Bibette J. 2000. Viscous Sintering Phenomena in LiquidLiquid Dispersions. Phys. Rev. Lett., 84(9): 2018-2021. DOI: https://doi.org/10.1103/PhysRevLett.84. 2018

Rehman K, Zulfakar MH. 2014. Recent advances in gel technologies for topical and transdermal drug delivery. Drug Development and Industrial Pharmacy, 40(4): $\quad 433-440 . \quad$ DOI: https://doi.org/10.3109/03639045.2013.8 28219

Rother M, Conaghan PG. 2013. A Randomized, Double-blind, Phase III Trial in Moderate Osteoarthritis Knee Pain Comparing Topical Ketoprofen Gel with Ketoprofen-free Gel. The Journal of Rheumatology, 40(10): 1742-1748. DOI: https://doi.org/10.3899/jrheum.130192

Sagiri SS, Behera B, Rafanan RR, Bhattacharya C, Pal K, Banerjee I, Rousseau D. 2014. Organogels as Matrices for Controlled Drug Delivery: A Review on the Current State. Soft Materials, 12(1): 47-72. DOI: https://doi.org/10.1080/1539445X.2012. 756016

Seidel EJ, Rother M, Regenspurger K, Rother I. 2015. A randomised trial comparing the efficacy and safety of topical ketoprofen in Transfersome gel (IDEA-033) with oral ketoprofen and drug-free ultradeformable Sequessome TM vesicles ( TDT 064 ) for the treatment of muscle soreness following exercise. Journal of Sports Sciences, 34(1): 88-95. DOI: https://doi.org/10.1080/02640414.2015.1 035667

Sy PM, Anton N, Gilet YI, Dieng SM, Messadeq N, Ennahar S, Diarra M, Vandamme T. 2018. Pickering nanoemulsion as a nanocarrier for $\mathrm{pH}-$ triggered drug release. International Journal of Pharmaceutics, 549(1-2): 299305.

DOI: 10.1016/j.ijpharm.2018.07.066

Verma A, Sukhdev S, Rupinder K, Ajay K, Upendra KJ. 2013. Formulation, Optimization And Evaluation Of Clobetasol Propionate Gel. International Journal of Pharmacy and Pharmaceutical Sciences, 5(4): 666-674. DOI: 10.1.1.954.9563 